\title{
A New Load Residual Threshold Definition for the Evaluation of Wind Tunnel Strain-Gage Balance Data
}

\author{
N. Ulbrich ${ }^{\dagger}$ and T. Volden $\ddagger$ \\ Jacobs Technology Inc., Moffett Field, California 94035
}

\begin{abstract}
A new definition of a threshold for the detection of load residual outliers of wind tunnel strain-gage balance data was developed. The new threshold is defined as the product between the inverse of the absolute value of the primary gage sensitivity and an empirical limit of the electrical outputs of a strain-gage. The empirical limit of the outputs is either $2.5 \mathrm{microV} / \mathrm{V}$ for balance calibration or check load residuals. A reduced limit of $0.5 \mathrm{microV} / \mathrm{V}$ is recommended for the evaluation of differences between repeat load points because, by design, the calculation of these differences removes errors in the residuals that are associated with the regression analysis of the data itself. The definition of the new threshold and different methods for the determination of the primary gage sensitivity are discussed. In addition, calibration data of a six-component force balance and a five-component semi-span balance are used to illustrate the application of the proposed new threshold definition to different types of strain-gage balances. During the discussion of the force balance example it is also explained how the estimated maximum expected output of a balance gage can be used to better understand results of the application of the new threshold definition.
\end{abstract}

\section{Nomenclature}

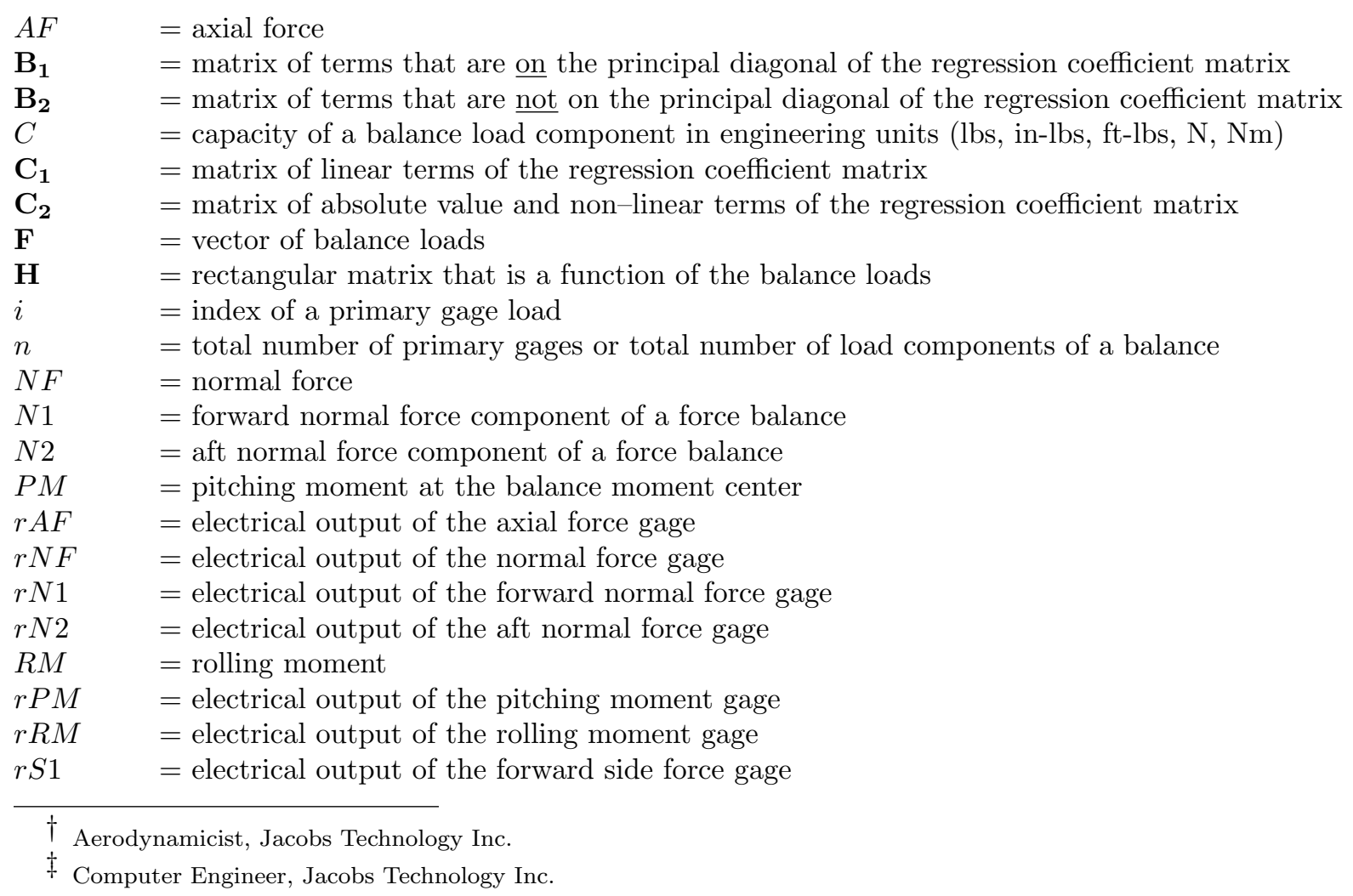




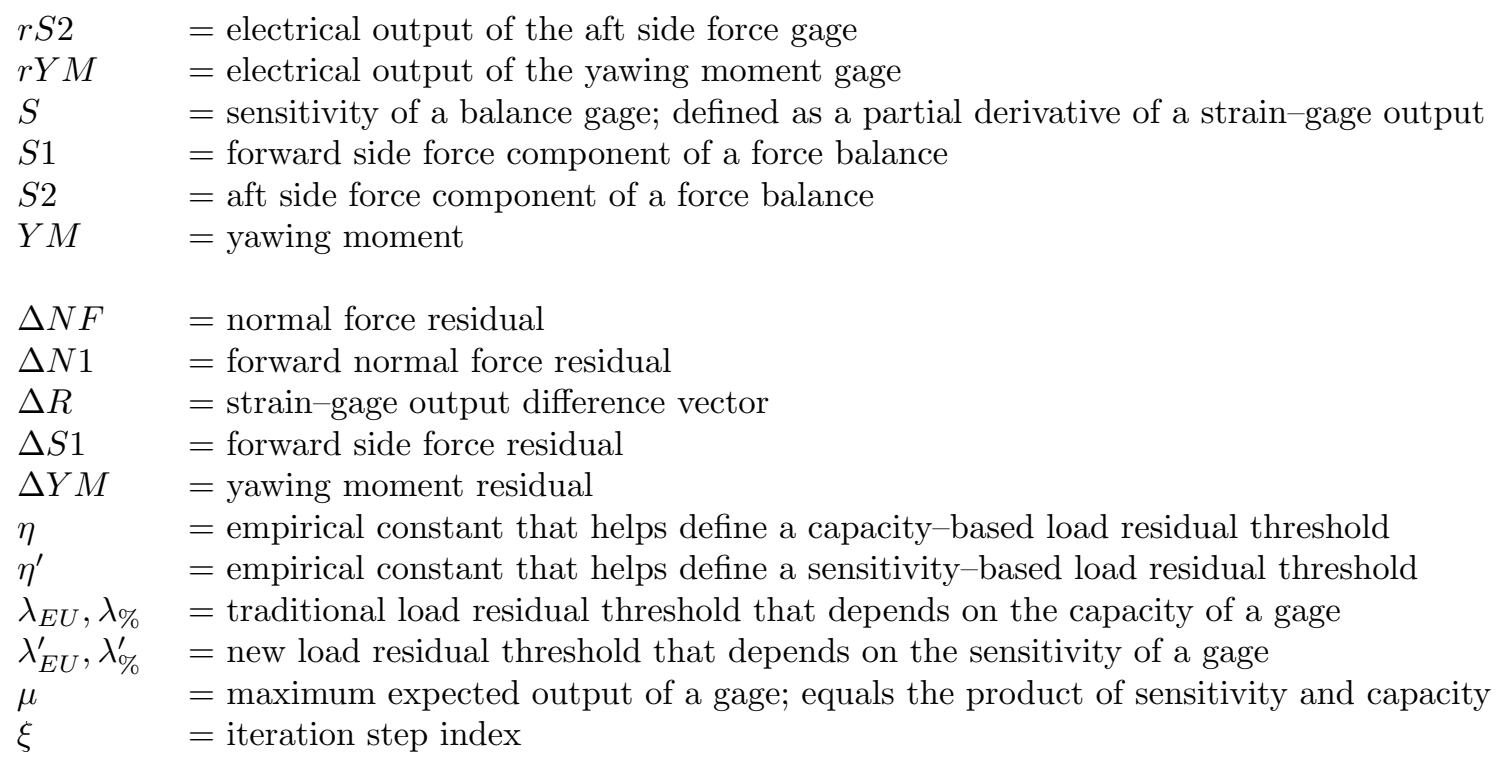

\section{Introduction}

In general, the accuracy of wind tunnel strain-gage balance data needs to be assessed in order to determine if (i) the calibration of the balance was successful, and if (ii) the balance will satisfy the customer's test goals. Load residuals are often used for this purpose. These load residuals are defined as the difference between the applied and predicted/fitted loads. They are a measure of load prediction errors that are the result of imperfections in data acquisition and regression analysis of the balance calibration data. Load residuals can be determined for both balance calibration and check load data.

Another important part of a comprehensive evaluation of strain-gage balance data is the assessment of the loads of repeat points. Predicted loads of repeat points have, of course, the same data acquisition and regression analysis errors as regular data points (including measurement uncertainty, noise, alignment errors, etc.). However, it is possible for repeat points to separate the data acquisition error from the regression analysis error. An analyst only has to compute the difference between the predicted load(s) of a repeat point and the arithmetic mean of the loads of the related repeat point set. In theory, the calculation of the repeat load difference removes the error associated with the regression analysis as this error is contained in both the load of the repeat point and the mean load of the related repeat point set.

Many analysts in the aerospace testing community use some small percentage of the load capacity of the gage to decide if a load residual meets their accuracy target, or, if it is a potential outlier. This traditional capacity-based residual threshold can be summarized as follows

TRADITIONAL RESIDUAL THRESHOLD (ENGINEERING UNITS)

$$
\begin{gathered}
\lambda_{E U}(i)= \pm \eta \times C(i) \\
\eta=\text { const. }=\left\{\begin{array}{lll}
0.0025 & \Longrightarrow \text { calibration/check load residuals } \\
0.0005 & \Longrightarrow \text { repeat load differences }
\end{array}\right.
\end{gathered}
$$

where $\eta$ is an empirical constant and $C(i)$ is the load capacity of gage $i$ expressed in engineering units (lbs, in-lbs, ft-lbs, N, or Nm). The residual threshold defined in Eq. (1a) assumes that the balance data is given in its "design" format (i.e., force balance in force balance format, or, moment balance in moment balance format, or, direct-read balance in direct-read format). Equation (1b) lists values of the empirical constant 
$\eta$ that are often used in the aerospace testing community. In general, the value of $\eta$ for the assessment of repeat load differences is significantly smaller than the corresponding value for calibration or check load residuals because the calculation of load differences of related repeat data points essentially removes errors associated with the least squares fit of the balance data.

It is useful to express the traditional threshold defined in Eq. (1a) as a percentage of the load capacity as load residuals are often plotted in that format. Then, the traditional threshold can be written as:

\section{TRADITIONAL RESIDUAL THRESHOLD (\% OF CAPACITY)}

$$
\lambda_{\%}(i)=\lambda_{E U}(i) \times \frac{100 \%}{C(i)}= \pm \eta \times 100 \%
$$

Equation (2) highlights another important characteristic of the traditional load residual threshold definition: the traditional threshold is the same for all balance gages if it is expressed as a percentage of the gage capacity. This conclusion can also be expressed as follows:

$$
\lambda_{\%}(i)=f(\eta) \text { and } \eta=\text { const. } \quad \Longrightarrow \quad \lambda_{\%}(1)=\ldots=\lambda_{\%}(i)=\ldots=\lambda_{\%}(n)
$$

The threshold characteristic described in Eq. (3) has a practical advantage: it is easy to apply because a fixed percentage of the gage capacity is used for all load components of the balance. This practical advantage, however, could also be interpreted as a disadvantage because the traditional threshold definition does not take into account if a specific strain-gage was optimally sized for the load range that is to be measured. Therefore, for example, the traditional threshold definition could lead an analyst into believing that a load residual of a data point exceeds the threshold even though, in reality, the residual is within its expected values. The manufacturer of the balance may have had to choose a gage with a lower sensitivity for the balance in order to satisfy some physical design constraints. It is also possible that an off-the-shelf gage with certain characteristics was not available for installation.

The authors decided to define a new load residual threshold that would address the shortcoming of the traditional threshold definition. First, the new load residual threshold definition is presented in the next section of the paper. Afterwards, data from the calibration of two different balances is used to illustrate benefits of the new threshold definition.

\section{Definition of New Load Residual Threshold}

It was pointed out in the previous section that the traditional load residual threshold definition has a disadvantage: it does not take the load resolution capability of each individual strain-gage into account. This important characteristic of a gage equals the product of the inverse of the gage sensitivity and the smallest detectable gage output change. Therefore, in order to address the shortcoming of the traditional threshold definition, the authors developed a new alternate definition that is based on the sensitivity.

The new load residual threshold definition can be better understood if the definition of the sensitivity itself is revisited. In general, the sensitivity of a sensor is described in the literature as follows: ... sensitivity ... is the ratio of a small change between constant output levels to the corresponding small change between constant input levels. ... (from Ref. [1], p. 39). In other words - the sensitivity of a balance gage can be described as the partial derivative of its electrical output with respect to the related primary gage load. Then, we can write:

\section{DEFINITION OF THE SENSITIVITY OF A GAGE}

$$
S \equiv \text { Sensitivity } \equiv \frac{\partial(\text { Primary Gage Output })}{\partial(\text { Primary Gage Load })}
$$


It is important to point out that Eq. (4) above is only valid if balance data is described in its design format. In that case, the output of each gage will primarily be caused by a single load component, i.e., the so-called "primary" gage load. In addition, the authors suggest to express the sensitivity in units of microV/V per engineering unit (lbs, in-lbs, ft-lbs, $\mathrm{N}, \mathrm{Nm}$ ) in order to make the metric both dimensionless and independent of the excitation voltage of the balance. This suggestion follows a general recommendation that is made in the literature (see Ref. [2], p. 48, Table 25). It should also be mentioned that the definition of the sensitivity is independent of the method that an analyst chooses for the analysis of balance calibration data. In other words - the definition is the same for both the Iterative and the Non-Iterative Method that may be used for the analysis (see, e.g., Ref. [3] for a detailed discussion of the two analysis methods).

The definition of the new sensitivity-based residual threshold is similar to the definition of the traditional threshold that is given in Eq. (1a). The capacity $C(i)$ on the right-hand side of Eq. $(1 a)$ is simply replaced by the inverse of the absolute value of the sensitivity $S(i)$. Then, the new threshold definition becomes

\section{NEW RESIDUAL THRESHOLD (ENGINEERING UNITS)}

$$
\begin{gathered}
\lambda_{E U}^{\prime}(i)= \pm \eta^{\prime} \times \frac{1}{|S(i)|} \\
\eta^{\prime}=\text { const. }= \begin{cases}2.5 \text { microV } / V & \Longrightarrow \text { calibration/check load residuals } \\
0.5 \text { microV } / V & \Longrightarrow \text { repeat load differences }\end{cases}
\end{gathered}
$$

where $\eta^{\prime}$ is a new empirical constant and $S(i)$ is the sensitivity of each gage expressed in units of microV/V per engineering unit. The new empirical constant $\eta^{\prime}$ has a useful "physical" interpretation. It is the "acceptable" dimensionless electrical output variation of a strain-gage. Its product with the inverse of a gage's sensitivity ultimately defines the new residual threshold. The authors suggest different levels of the constant $\eta^{\prime}$ that depend on the type of residual under investigation. The constant ranges from $0.5 \mathrm{microV} / \mathrm{V}$ for repeat load differences to 2.5 microV $/ V$ for calibration or check load residuals.

Again, it is useful to express the new sensitivity-based residual threshold as a percentage of the load capacity. Then, the new threshold can be written as

\section{NEW RESIDUAL THRESHOLD (\% OF CAPACITY)}

$$
\begin{gathered}
\lambda_{\%}^{\prime}(i)=\lambda_{E U}^{\prime}(i) \times \frac{100 \%}{C(i)}= \pm \frac{\eta^{\prime}}{\mu(i)} \times 100 \% \\
\mu(i)=|S(i)| \times C(i) \equiv \text { maximum expected output of the gage }
\end{gathered}
$$

The new threshold has an important property when compared with the traditional threshold that is described in Eq. (2). The new threshold is no longer identical for all balance gages if it is expressed as a percentage of the capacity. This conclusion can also be summarized as follows:

$$
\lambda_{\%}^{\prime}(i)=f\left(\eta^{\prime}, \mu(i)\right) \text { and } \mu(i) \neq \text { const. } \quad \Longrightarrow \quad \lambda_{\%}^{\prime}(1) \neq \ldots \neq \lambda_{\%}^{\prime}(i) \neq \ldots \neq \lambda_{\%}^{\prime}(n)
$$

Equation (7) highlights the fact that the new threshold is a function of both the sensitivity and the capacity of each individual gage if it is expressed as a percentage of the gage capacity. Calibration data sets of two different types of strain-gage balances are used in the next section of the paper to illustrate the application of the new load residual threshold definition to experimental data. 


\section{Application of New Threshold to Balance Data}

\section{A. General Remarks}

Calibration data of two strain-gage balances were selected to demonstrate the application of the new load residual threshold. The first balance is NASA's MC60E six-component force balance. The balance was calibrated using Triumph Aerospace's Automatic Balance Calibration System (ABCS) in 2008. The calibration data set consists of 1904 loadings (no repeat loads were applied during the calibration). The second balance is NASA's MC400 five-component semi-span balance that was calibrated in Triumph Aerospace's Large Load Rig (LLR) in 2012. This calibration data set has a total of 1282 data points distributed across 93 load series. A subset of 432 data points of the 1282 points were repeats.

The analysis of the two selected balance calibration data sets was performed using NASA's BALFIT regression analysis tool (cf. Ref. [4]). It was critical during the analysis of the two data sets to apply BALFIT's regression model term reduction algorithm. This approach prevented unwanted "over-fitting" of the two data sets that would artificially decrease the magnitude of the residuals by including statistically insignificant terms in the regression models of the outputs. The determination of the primary gage sensitivities of a balance is reviewed in the next section of the paper because they are needed for the definition of the new threshold. Afterwards, results of the analysis of the two balance data sets are discussed.

\section{B. Determination of the Primary Gage Sensitivity}

Most balances are designed such that each load component, i.e., each primary gage load, can directly be associated with one gage output, i.e., the related primary gage output. Therefore, assuming that (i) the Iterative Method is used to process balance data in the least squares sense and that (ii) the data is given in its design format, the regression coefficient of the linear term of the primary gage load often explains about $90 \%$ (or more) of the total electrical output of the related primary gage. This regression coefficient equals the "sensitivity" of the balance gage. It is typically expressed in units of microV/V divided by the unit of the related primary gage load. It can directly be copied from the "regression coefficient matrix" (also called "calibration" or "curve-fit" matrix in the literature). It is important to emphasize again that the above description of the determination of the "sensitivity" is only valid if the balance is analyzed in its design format. Otherwise, it is not possible to uniquely associate one primary load with one primary gage output and, consequently, the "sensitivity" cannot be defined.

Often, an analyst provides the "data reduction matrix" instead of the "regression coefficient matrix" to the end user of the balance because coefficients contained in the "data reduction matrix" directly describe the load iteration scheme that is needed to predict loads from gage outputs during a wind tunnel test (see, e.g., Refs. [2] and [5] for a detailed discussion of the "data reduction matrix" and the associated load iteration schemes). In this situation, the end user can still "reverse-engineer" the missing "exact" values of the "sensitivities" from the coefficients of the "data reduction matrix." The "data reduction matrix" itself may be generated for two different types of load iteration schemes that are historically being used in the aerospace testing community (equations of the two load iteration schemes are listed in Table 1 below).

Table 1: Load iteration equation choices for the Iterative Method.

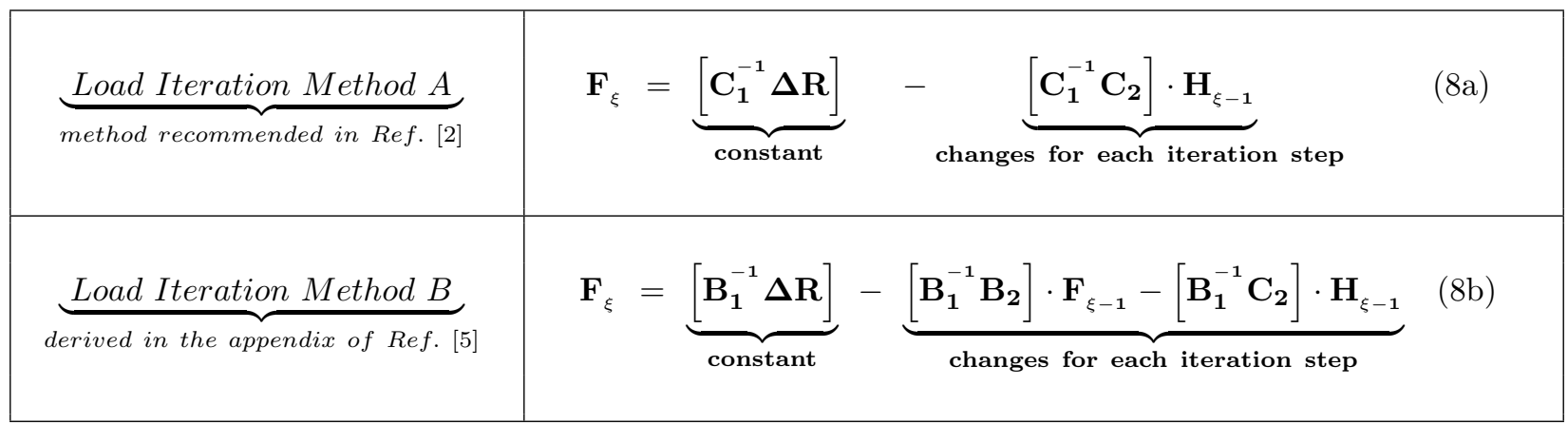

The following symbols are used in Table 1 above: (i) the vector $\mathbf{F}_{\xi}$ represents the loads for each iteration 
step with index $\xi$, (ii) $\Delta R$ are the electrical output changes of the balance gages, and (iii) $\mathbf{C}_{\mathbf{1}}^{-1}, \mathbf{C}_{\mathbf{2}}, \mathbf{B}_{\mathbf{1}}^{-1}$, and $\mathbf{B}_{\mathbf{2}}$ are fixed square matrices, and (iv) $\mathbf{H}_{\xi-1}$ is a rectangular matrix that depends on vector $\mathbf{F}_{\xi-1}$ and is updated after completion of each iteration step. The end user of a balance may simply use the inverse values of the coefficients on the principle diagonal of either square matrix $\mathbf{C}_{\mathbf{1}}^{-1}$ (Load Iteration Method A) or square matrix $\mathbf{B}_{\mathbf{1}}^{-1}$ (Load Iteration Method B) as the missing "sensitivities."

It is important to point out that only the inverse values of the coefficients on the principle diagonal of square matrix $\mathbf{B}_{1}^{-1}$ will precisely match the "exact" values of the "sensitivities" from the original "regression coefficient matrix" because, by design, all off-diagonal elements of matrix $\mathbf{B}_{1}^{-1}$ are zero. This characteristic is an advantage of Load Iteration Method B.

The inverse values of the coefficients on the principle diagonal of square matrix $\mathbf{C}_{\mathbf{1}}^{-1}$, on the other hand, are close "approximations" of the "exact" values of the "sensitivities" because the off-diagonal elements of matrix $\mathbf{C}_{1}^{-1}$ are not necessarily all zero. Therefore, the authors recommend users of Load Iteration Method A to always directly copy sensitivities from the "regression coefficient matrix", i.e., from the original regression models of the fitted gage outputs, instead of "reverse-engineering" sensitivities from the data reduction matrix coefficients that are contained in square matrix $\mathbf{C}_{1}^{-1}$.

\section{MC60E Six-Component Force Balance}

Data from the calibration of NASA's MC60E balance is used in this section to illustrate the application of the new load residuals threshold definition. The MC60E is a 2.0 inch diameter multi-piece force balance with characteristics that are very similar to those of a single-piece balance. Therefore, no absolute value terms are needed in regression models of its gage outputs. Table 2 below lists load capacities of the MC60E balance in force balance format.

Table 2: Load capacities of NASA's MC60E six-component force balance.

\begin{tabular}{|c|c|c|c|c|c|}
\hline$N 1, \mathrm{lbs}$ & $N 2, \mathrm{lbs}$ & $S 1, \mathrm{lbs}$ & $S 2, \mathrm{lbs}$ & $R M$, in-lbs & $A F, \mathrm{lbs}$ \\
\hline 2,500 & 2,500 & 1,250 & 1,250 & 5,000 & 700 \\
\hline
\end{tabular}

BALFIT's regression model term reduction algorithm was applied during the analysis of the MC60E data set. Figure 1 shows the final optimized regression models that were used to fit the outputs of the $\mathrm{MC60E}$ balance. Each column describes the regression model of an output. Black rectangles mark the chosen regression model terms. Figure $2 \mathrm{a}$ lists a subset of the regression coefficients of the math model for each output (only coefficients of the first seven terms are shown). Blue boxes mark coefficient values in Fig. 2a that are the "exact" values of the primary gage sensitivities of the six balance gages.

The authors decided to transform the partially displayed regression coefficient matrix of Fig. 2a to the corresponding data reduction matrix by using both the matrix format of Load Iteration Method $A$ and the matrix format of Load Iteration Method B. Consequently, the previously discussed differences between reverse-engineered "exact" and "approximate" values of the sensitivities can be illustrated by using the calibration data of the MC60E balance. Figure $2 \mathrm{~b}$ shows a subset of the data reduction matrix coefficients for Load Iteration Method A. Only the intercept and coefficients of matrix $\mathbf{C}_{\mathbf{1}}^{-\mathbf{1}}$ are shown. Similarly, Fig. 2c shows a subset of the data reduction matrix coefficients for Load Iteration Method B. Again, only the intercept and coefficients of matrix $\mathbf{B}_{1}^{-1}$ are shown. Red boxes mark coefficient values on the principle diagonal of matrix $\mathbf{C}_{\mathbf{1}}^{-\mathbf{1}}$ (Fig. 2b) and matrix $\mathbf{B}_{\mathbf{1}}^{-\mathbf{1}}$ (Fig. 2c).

It was mentioned at the end of the previous section that the inverse values of the coefficients on the principle diagonal of matrix $\mathbf{C}_{\mathbf{1}}^{-1}$ are close "approximations" of the "exact" values of the "sensitivities". It was also pointed out that the inverse values of the coefficients on the principle diagonal of matrix $\mathbf{B}_{\mathbf{1}}^{-1}$ precisely match the "exact" values of the "sensitivities". These two assertions can easily be illustrated using the coefficients that are shown in Figs. 2a, 2b, and 2c. The table in Fig. 2d summarizes corresponding values. It can be seen in Fig. 2d that the "exact" sensitivities from Fig. 2a and the "reverse-engineered" sensitivities from Fig. 2c show "perfect" agreement. The "reverse-engineered" sensitivities from Fig. 2b, on the other hand, are close "approximations" of the "exact" sensitivities from Fig. 2a.

The calibration load residuals of the forward normal and forward side force component were compared 
in the next step by using the traditional and the new load residual threshold definitions. Figure 3a shows residuals of the two load components if they are assessed by using the fixed traditional residual threshold of $0.25 \%$ of capacity. Figure $3 \mathrm{~b}$ shows residuals of the two load components if they are assessed by using the new load residual threshold definition. In that case, the threshold varies gage by gage and is no longer a constant percentage of the capacity. The threshold value for the forward normal force component equals $0.236 \%$ of capacity. The threshold value for the forward side force component equals $0.435 \%$ of capacity. It can be seen, after comparing the plot of the forward side force component in Fig. 3a with the corresponding plot in Fig. 3b, that the new threshold definition has increased the threshold from the fixed value of $0.25 \%$ to the elevated value of $0.435 \%$. Consequently, fewer data points are potential "outliers" as the sensitivity of the forward side force gage was used to define the threshold.

The root cause of the difference between the overall level of the load residuals for the forward normal force $(N 1)$ and the forward side force $(S 1)$ can be investigated in more detail. Therefore, the maximum expected output of the balance gages was computed. This useful metric was defined in Eq. (6b) above. Table 3 below shows the corresponding maximum expected outputs for the six gages of the MC60E balance.

Table 3: Maximum expected output $\mu(i)$ of the six gages of NASA's MC60E balance.

\begin{tabular}{|c|c|c|c|c|c|}
\hline $\begin{array}{c}r N 1 \\
\mathrm{microV} / \mathrm{V}\end{array}$ & $\begin{array}{c}r N 2 \\
\text { microV/V }\end{array}$ & $\begin{array}{c}r S 1 \\
\text { microV/V }\end{array}$ & $\begin{array}{c}r S 2 \\
\mathrm{microV} / \mathrm{V}\end{array}$ & $\begin{array}{c}r R M \\
\mathrm{microV} / \mathrm{V}\end{array}$ & $\begin{array}{c}r A F \\
\mathrm{microV} / \mathrm{V}\end{array}$ \\
\hline 1059 & 1092 & 575 & 618 & 1362 & 819 \\
\hline
\end{tabular}

It is observed, after comparing the maximum expected output of the forward normal force gage $(1059$ microV $/ V)$ with the maximum expected output of the forward side force gage $(575$ microV/V) that the values differ significantly. It is the authors' experience that a well matched gage of a force balance has a maximum expected output of $\approx 1000 \mathrm{microV} / \mathrm{V}$. Therefore, it appears that the balance designers may have had to make compromises as far as balance size, flexure design, gage placement, and gage selection are concerned that resulted in a reduced maximum expected output for the forward side force gage.

\section{MC400 Five-Component Semi-span Balance}

Data from a calibration of NASA's MC400 balance is used in this section to illustrate the application of the new threshold definition to another balance type. The MC400 is a five-component semi-span balance that was calibrated in 2012 in Triumph Aerospace's Large Load Rig. The balance is a single-piece design. Therefore, no absolute value terms are needed in the regression models of its outputs. Table 4 below lists capacities of the MC400 in direct-read format. It is the original design format of the balance.

Table 4: Load capacities of NASA's MC400 five-component semi-span balance.

\begin{tabular}{|c|c|c|c|c|}
\hline$N F$, lbs & $P M$, in-lbs & $Y M$, in-lbs & $R M$, in-lbs & $A F$, lbs \\
\hline 40,000 & 240,000 & 480,000 & $2,300,000$ & 8,000 \\
\hline
\end{tabular}

First, calibration load residuals of the normal force and yawing moment were compared by using the traditional and new threshold definitions. Figure 4a shows residuals of the two load components if they are compared against the fixed traditional threshold of $0.25 \%$ of capacity. Figure $4 \mathrm{~b}$ shows residuals of the two load components if they are compared against the new threshold definition. The threshold for the normal force equals $0.280 \%$ of capacity. The threshold for the yawing moment equals $0.302 \%$ of capacity. The new threshold values of the two load components are very close in magnitude. This observation can be understood if the maximum expected outputs of the gages are compared. Table 5 below lists these values.

Table 5: Maximum expected output $\mu(i)$ of the five gages of NASA's MC400 balance.

\begin{tabular}{|c|c|c|c|c|}
\hline $\begin{array}{c}r N F \\
\mathrm{microV} / \mathrm{V}\end{array}$ & $\begin{array}{c}r P M \\
\mathrm{microV} / \mathrm{V}\end{array}$ & $\begin{array}{c}r Y M \\
\mathrm{microV} / \mathrm{V}\end{array}$ & $\begin{array}{c}r R M \\
\mathrm{microV} / \mathrm{V}\end{array}$ & $\begin{array}{c}r A F \\
\mathrm{microV} / \mathrm{V}\end{array}$ \\
\hline 894 & 1191 & 829 & 780 & 636 \\
\hline
\end{tabular}


It is observed, after comparing the maximum expected output of the normal force gage (894 microV/V) with the maximum expected output of the yawing moment gage $(829 \mathrm{microV} / \mathrm{V})$ that the values are close to the maximum expected output of a well matched gage $(\approx 1000 \mathrm{microV} / \mathrm{V})$. This results indicates that the normal and yawing moment gages of the MC400 appear to match corresponding load capacities well.

The calibration data set of the MC400 balance consisted of 1282 points. A subset of 432 of the 1282 data points were repeat loads. Therefore, it is possible to compare the repeat load differences of the MC400 against the new residual threshold. Two threshold options were used to assess the repeat load differences of the normal force and the yawing moment: (i) the fixed "traditional" threshold of $0.05 \%$ of capacity and (ii) the new residual threshold with $\eta^{\prime}=0.5 \mathrm{microV} / \mathrm{V}$ as input. Figure 5 a compares the residual assessment of the repeat loads of the two load components for the traditional threshold. Figure $5 \mathrm{~b}$ compares the residual assessment of the repeat loads of the two load components for the new threshold. The residuals were plotted in both cases versus the original data point index. It appears, after comparing the assessment of the repeat load differences for the two load components using the two threshold options, that the new residual threshold definition is also suited to identify potential repeat load difference outliers.

\section{Summary and Conclusions}

A new load residual threshold definition was discussed that may be used to better evaluate wind tunnel strain-gage balance data. The new threshold definition uses the sensitivity instead of the capacity of each individual balance gage as input. Consequently, it provides a more realistic assessment of the performance of a data point as the load resolution capability of each individual balance gage is taken into account.

Recommendations related to the numerical determination of the sensitivity of a balance gage were made that consider an analyst's load iteration method choice. In addition, data from the calibration of NASA's MC60E six-component force balance and NASA's MC400 five-component semi-span balance were used to illustrate the application of the new residual threshold definition to both calibration load residuals and repeat load differences.

\section{Acknowledgements}

The authors want to thank Bob Gisler of NASA Ames Research Center for his critical and constructive review of the final manuscript. The work reported in this paper was supported by the Wind Tunnel Division at NASA Ames Research Center under contract NNA09DB39C.

\section{References}

${ }^{1}$ Draper, C. S., McKay, W., Lees, S., Instrumentation Engineering, Vol. 1, McGraw-Hill Book Company, Inc., New York, Toronto, London, 1952; pp. 39-40.

${ }^{2}$ American Institute of Aeronautics and Astronautics, Recommended Practice - Calibration and Use of Internal Strain Gage Balances with Application to Wind Tunnel Testing, AIAA R-091-2003, American Institute of Aeronautics and Astronautics, Reston, Virginia, 2003; pp. 11-19, p. 48, Table 25.

${ }^{3}$ Ulbrich, N., "Comparison of Iterative and Non-Iterative Strain-Gage Balance Load Calculation Methods," AIAA 2010-4202, paper presented at the 27th AIAA Aerodynamic Measurement Technology and Ground Testing Conference, Chicago, Illinois, June/July 2010.

${ }^{4}$ BALFIT - Software Tool for the Regression Analysis of Multivariate Data - Version 3.01.21, distributed by the Software Release Authority, Technology Partnerships Office, NASA Ames Research Center, Moffett Field, California, December 2014.

${ }^{5}$ Ulbrich, N., and Volden, T., "Application Of A New Calibration Analysis Process to the MK-III-C Balance," AIAA 2006-0517, paper presented at the 44th AIAA Aerospace Sciences Meeting, Reno, Nevada, January 2006. 


$$
\begin{gathered}
\text { NUMBER OF TERMS }=15,25,18,23,17,18 \\
\text { HIERARCHICAL: rN1, rN2, rS1, rS2, rRM, rAF }
\end{gathered}
$$

(HIERARCHY ANALYSIS USES $|F * G|=|F| *|G|,|F * F * F|=|F| *|F| *|F|,|F| *|F|=F * F$ )
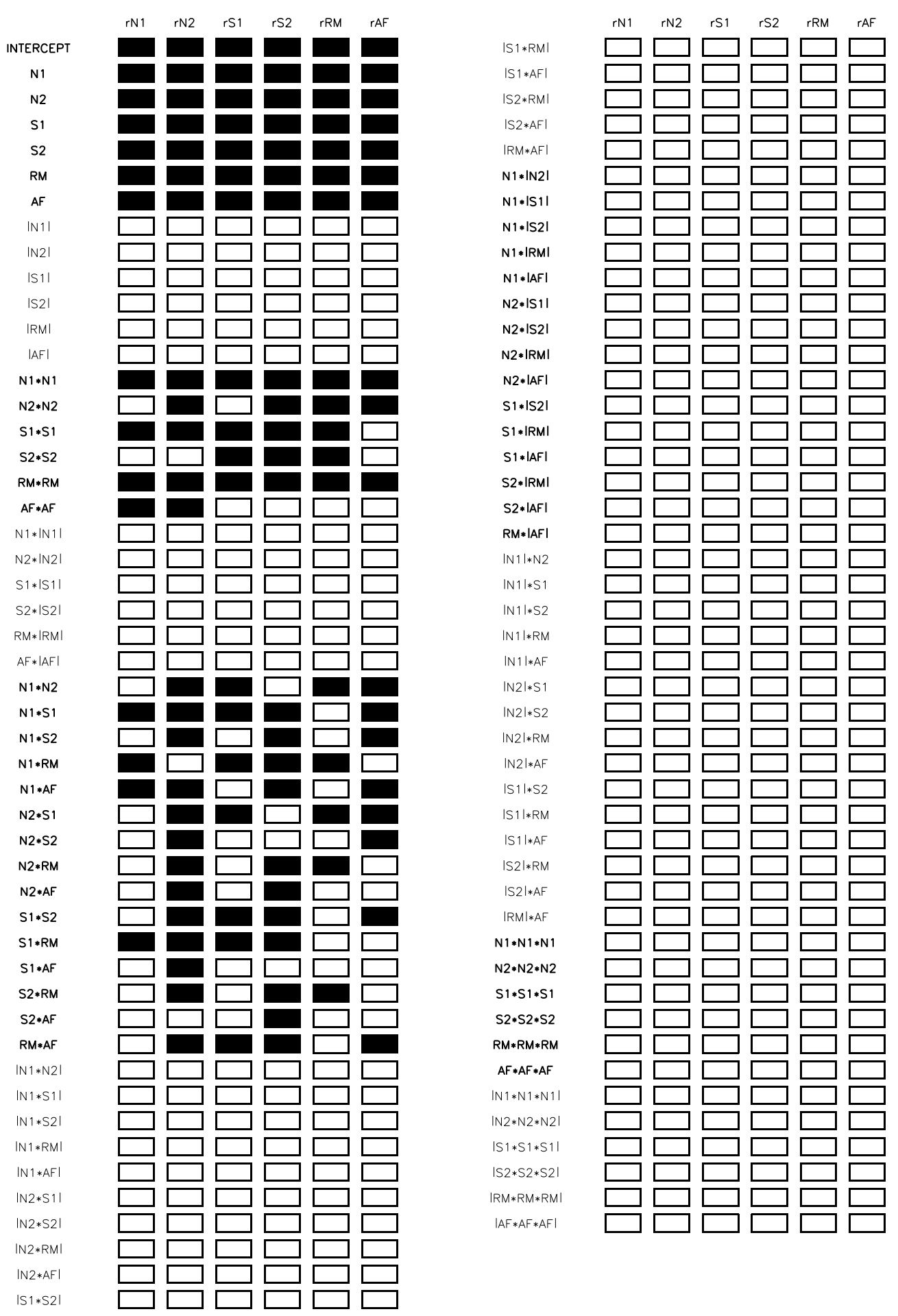

Fig. 1 Optimized regression models used for the analysis of the MC60E balance calibration data. 


\begin{tabular}{|c|c|c|c|c|c|c|c|}
\hline INDEX & TERM & $\mathrm{rN} 1$ & $\mathrm{rN} 2$ & rS1 & rS2 & rRM & $\mathrm{rAF}$ \\
\hline 1 & INTERCEPT & $-6.677237 e-02$ & $+4.212307 e-01$ & $-5.150053 e-01$ & $+1.767285 e-01$ & $+1.113018 \mathrm{e}+00$ & $-1.759241 e-01$ \\
\hline 2 & N1 & $+4.235624 \mathrm{e}-01$ & $+3.011417 e-02$ & $-1.920499 e-02$ & $+6.538245 e-02$ & $+2.461057 e-02$ & $+7.228774 \mathrm{e}-03$ \\
\hline 3 & N2 & $-1.229270 e-03$ & $+4.366356 \mathrm{e}-01$ & $+9.007268 e-04$ & $-8.752121 e-03$ & $+1.318943 e-02$ & $+7.805780 e-04$ \\
\hline 4 & S1 & $-1.884619 e-02$ & $+7.377303 e-02$ & $+4.601742 e-01$ & $+2.263832 \mathrm{e}-02$ & $+3.299738 \mathrm{e}-02$ & $-1.930152 e-04$ \\
\hline 5 & S2 & $-4.025318 e-03$ & $+1.586084 \mathrm{e}-03$ & $-9.806925 e-04$ & $+4.947825 e-01$ & $+6.788265 e-03$ & $-1.678125 e-03$ \\
\hline 6 & RM & $-1.941069 e-03$ & $+3.975029 e-02$ & $+6.116269 e-03$ & $+3.664388 \mathrm{e}-02$ & $+2.722102 e-01$ & $+7.460587 e-03$ \\
\hline 7 & $\mathrm{AF}$ & $+1.954011 \mathrm{e}-02$ & $-9.993063 e-02$ & $+5.199735 e-02$ & $+7.763641 \mathrm{e}-02$ & $-1.013342 e-02$ & $+1.169995 e+00$ \\
\hline
\end{tabular}

Fig. 2a Regression coefficient matrix of the optimized models of the MC60E calibration data (only coefficients of the intercept and the six primary load components are shown).

\begin{tabular}{|c|c|c|c|c|c|c|c|}
\hline INDEX & TERM & N1 & N2 & S1 & S2 & $\mathrm{RM}$ & $\mathrm{AF}$ \\
\hline 1 & INTERCEPT & $-6.677237 e-02$ & $+4.212307 e-01$ & $-5.150053 e-01$ & $+1.767285 e-01$ & $+1.113018 \mathrm{e}+00$ & $-1.759241 e-01$ \\
\hline- & $\mathrm{rN} 1$ & $+2.361807 e+00$ & $-1.630148 e-01$ & $+1.025803 e-01$ & $-3.019185 e-01$ & $-2.110431 e-01$ & $-1.355397 e-02$ \\
\hline- & rN2 & $+6.541463 e-03$ & $+2.300145 e+00$ & $-2.535727 e-03$ & $+4.842837 e-02$ & $-1.129702 e-01$ & $-7.855816 e-04$ \\
\hline- & rS1 & $+9.383080 e-02$ & $-3.511547 e-01$ & $+2.180679 e+00$ & $-9.986181 e-02$ & $-2.532655 e-01$ & $+1.486037 \mathrm{e}-03$ \\
\hline- & rS2 & $+1.905915 e-02$ & $-4.087865 e-03$ & $+5.456845 e-03$ & $+2.021647 e+00$ & $-5.248539 e-02$ & $+3.120201 \mathrm{e}-03$ \\
\hline- & rRM & $+1.242410 e-02$ & $-3.344224 e-01$ & $-4.590042 e-02$ & $-2.757584 e-01$ & $+3.700266 e+00$ & $-2.385186 e-02$ \\
\hline- & rAF & $-4.421304 e-02$ & $+2.121616 \mathrm{e}-01$ & $-9.960399 e-02$ & $-1.229204 e-01$ & $+4.066248 \mathrm{e}-02$ & $+8.543844 e-01$ \\
\hline
\end{tabular}

Fig. 2 b Data reduction matrix of the optimized models of the MC60E calibration data (only coefficients of matrix $\mathbf{C}_{\mathbf{1}}^{-1}$ of Load Iteration Method A are shown).

\begin{tabular}{|c|c|c|c|c|c|c|c|}
\hline INDEX & TERM & $\mathrm{N} 1$ & N2 & S1 & S2 & $\mathrm{RM}$ & AF \\
\hline 1 & INTERCEPT & $-6.677237 e-02$ & $+4.212307 \mathrm{e}-01$ & $-5.150053 e-01$ & $+1.767285 \mathrm{e}-01$ & $+1.113018 \mathrm{e}+00$ & $-1.759241 e-01$ \\
\hline- & $\mathrm{rN} 1$ & $+2.360927 \mathrm{e}+00$ & 0 & 0 & 0 & 0 & 0 \\
\hline- & $\mathrm{rN} 2$ & 0 & $+2.290239 \mathrm{e}+00$ & 0 & 0 & 0 & 0 \\
\hline- & rS1 & 0 & 0 & $+2.173090 \mathrm{e}+00$ & 0 & 0 & 0 \\
\hline- & rS2 & 0 & 0 & 0 & $+2.021090 \mathrm{e}+00$ & 0 & 0 \\
\hline- & rRM & 0 & 0 & 0 & 0 & $+3.673632 \mathrm{e}+00$ & 0 \\
\hline- & rAF & 0 & 0 & 0 & 0 & 0 & $+8.547048 \mathrm{e}-01$ \\
\hline
\end{tabular}

Fig. 2c Data reduction matrix of the optimized models of the MC60E calibration data (only coefficients of matrix $\mathbf{B}_{\mathbf{1}}^{-1}$ of Load Iteration Method B are shown).

American Institute of Aeronautics and Astronautics 


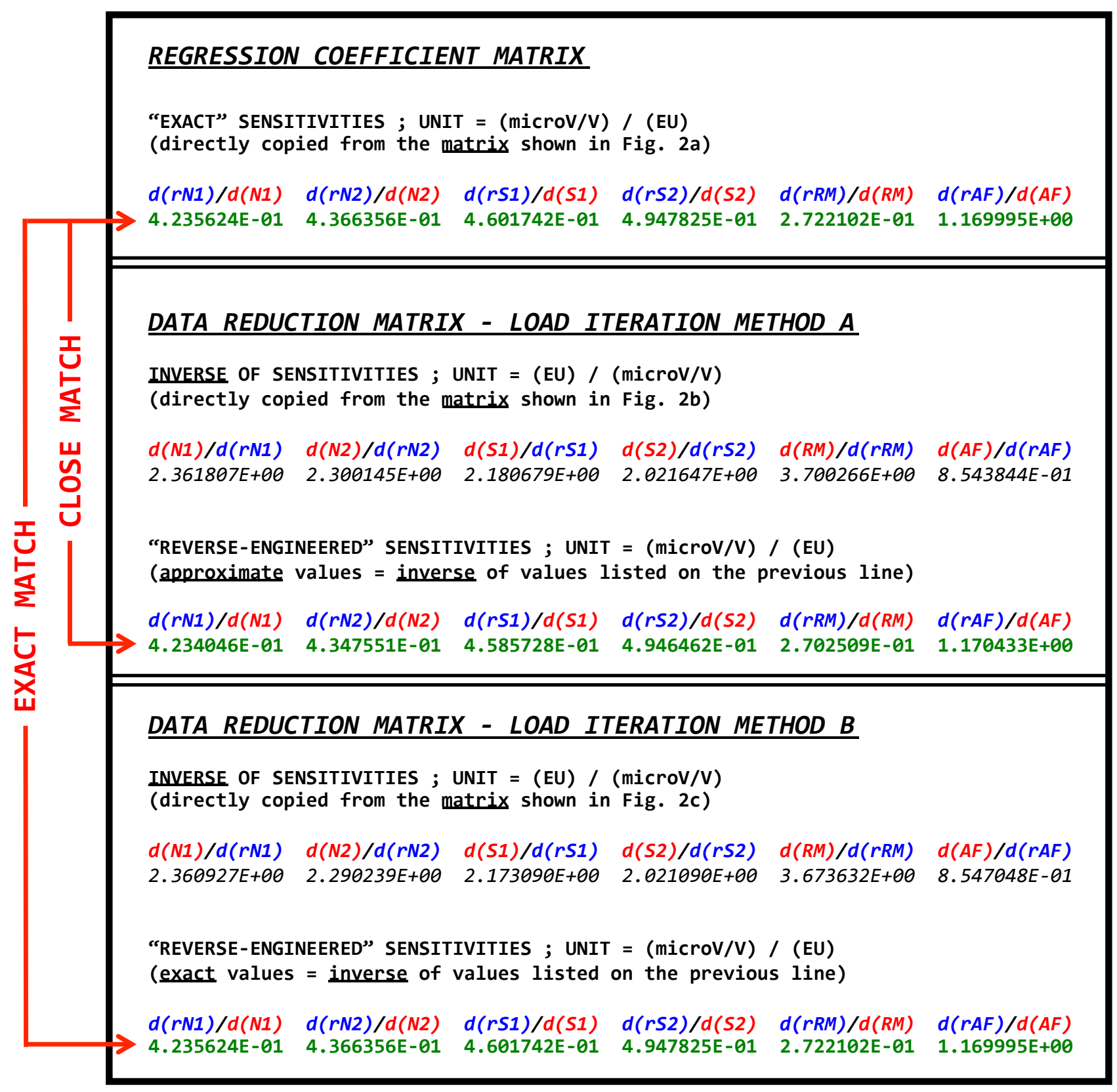

Fig. 2d Table of "exact" and "approximate" values of the primary gage sensitivities of the MC60E. 

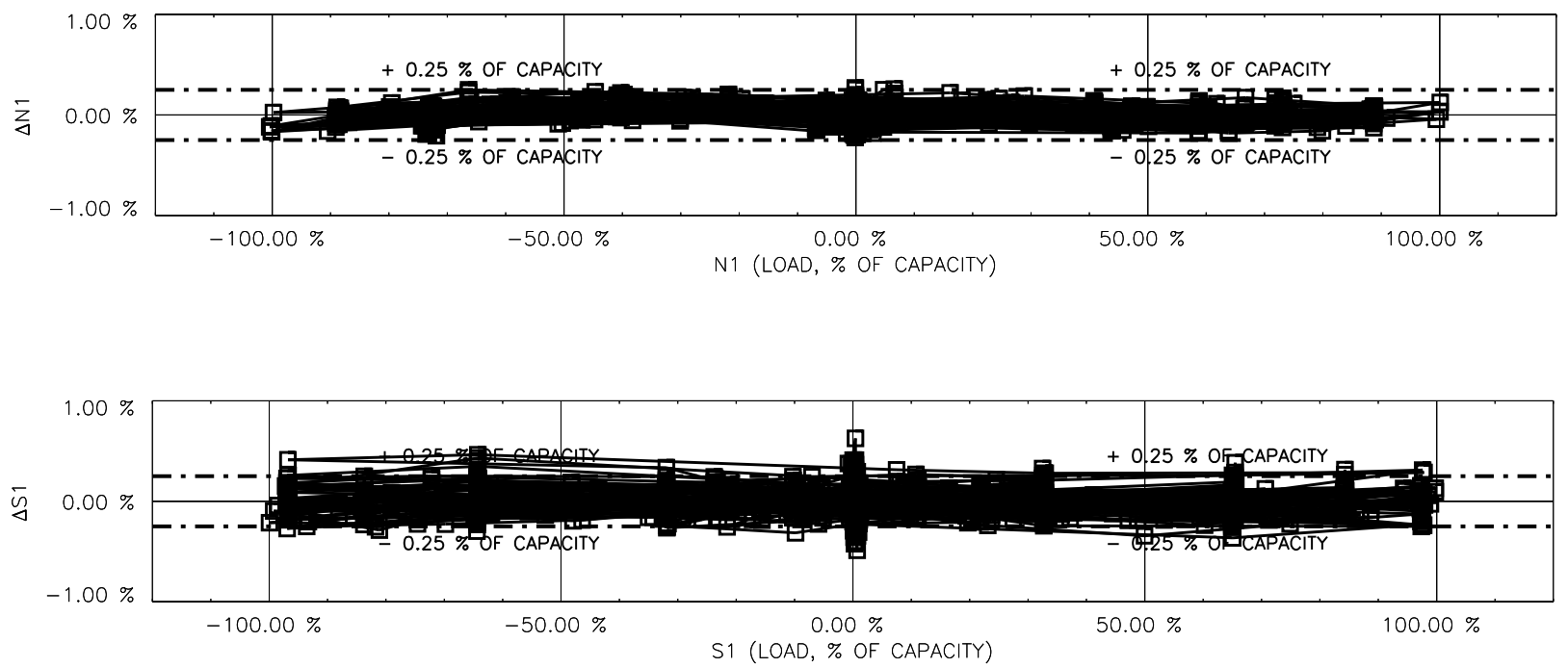

Fig. 3a Forward normal and side force residuals for the MC60E force balance calibration data (load residual threshold $= \pm 0.25 \%$ of the load capacity).
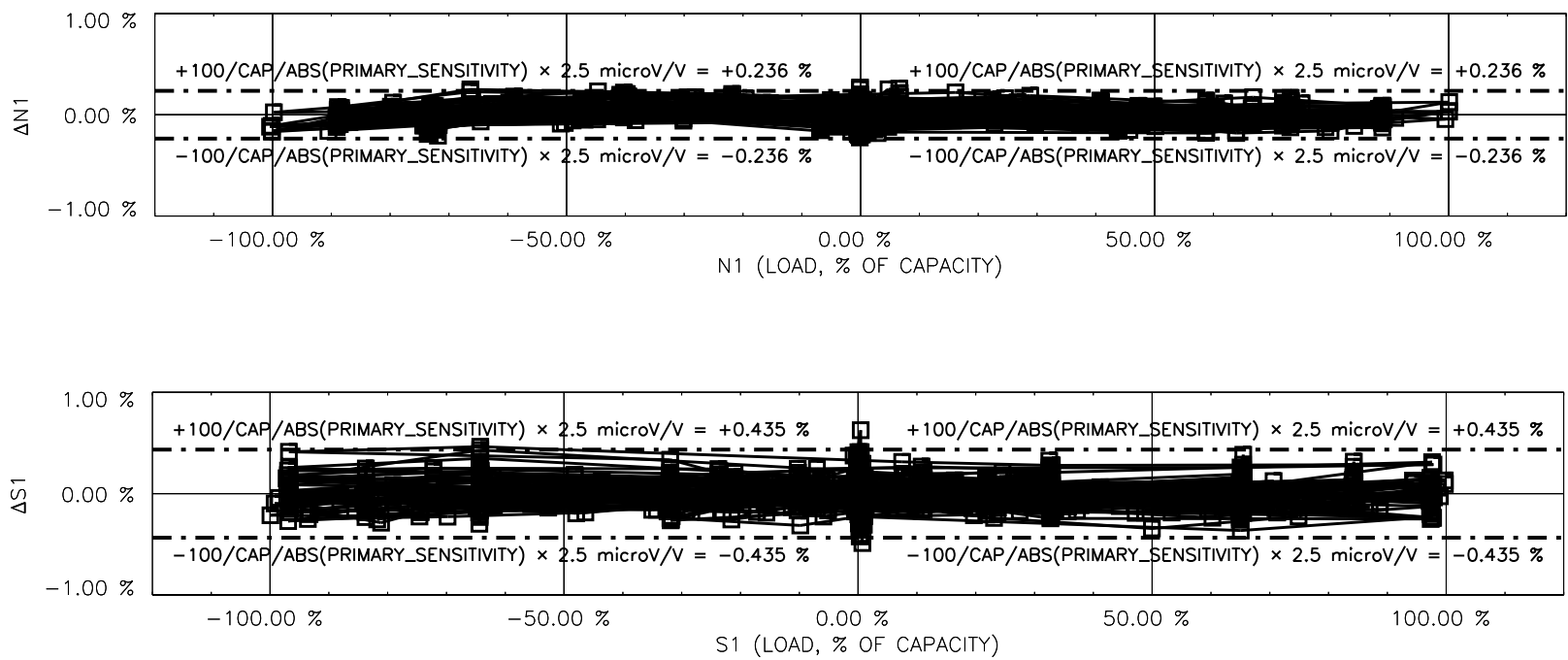

Fig. 3b Forward normal and side force residuals for the MC60E force balance calibration data (load residual threshold $= \pm 100 /$ capacity $/ \mid$ sensitivity $\mid \times 2.5$ microV $/ V$ ). 

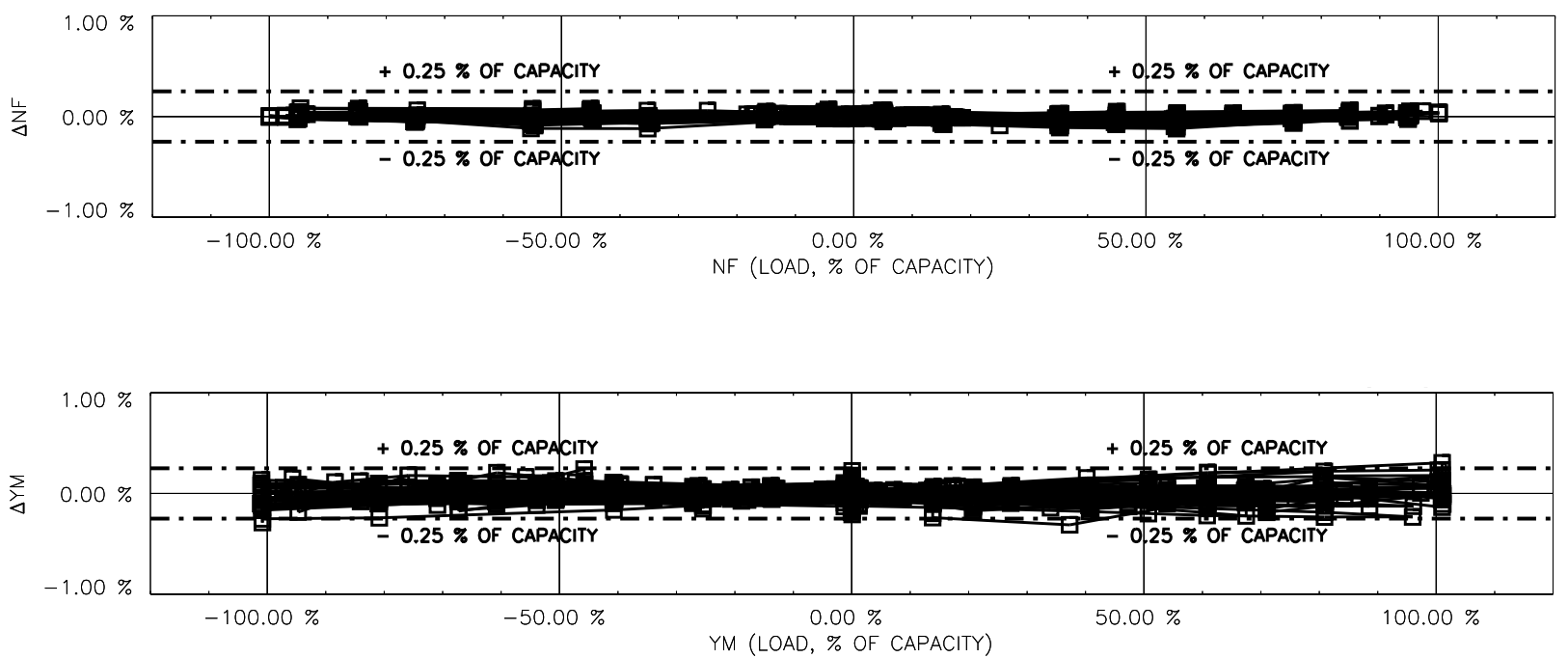

Fig. 4a Normal force and yawing moment residuals for the MC400 balance calibration data (load residual threshold $= \pm 0.25 \%$ of the load capacity).
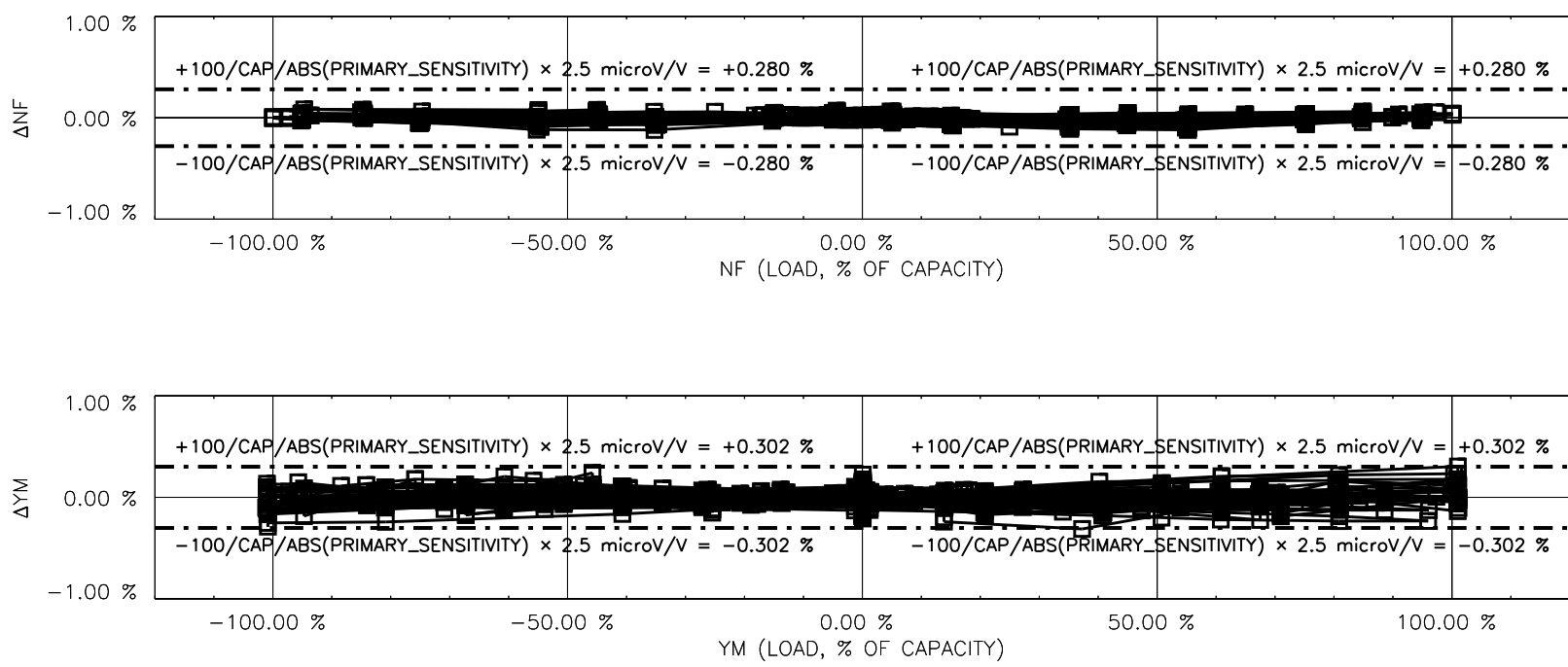

Fig. 4b Normal force and yawing moment residuals for the MC400 balance calibration data (load residual threshold $= \pm 100 /$ capacity $/ \mid$ sensitivity $\mid \times 2.5$ microV $/ V$ ). 

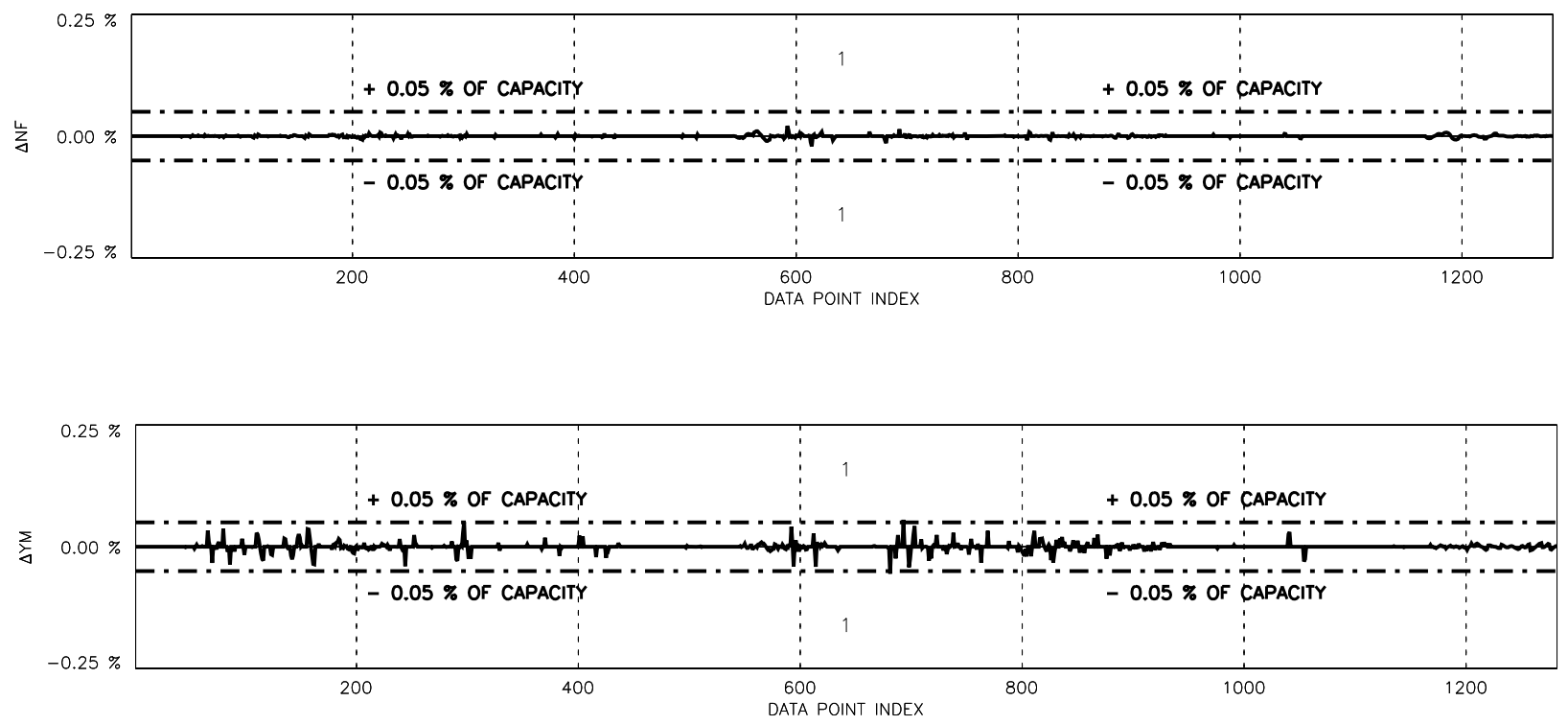

Fig. 5a Normal force and yawing moment repeat load differences for the MC400 balance calibration data (repeat load difference threshold $= \pm 0.05 \%$ of the load capacity).
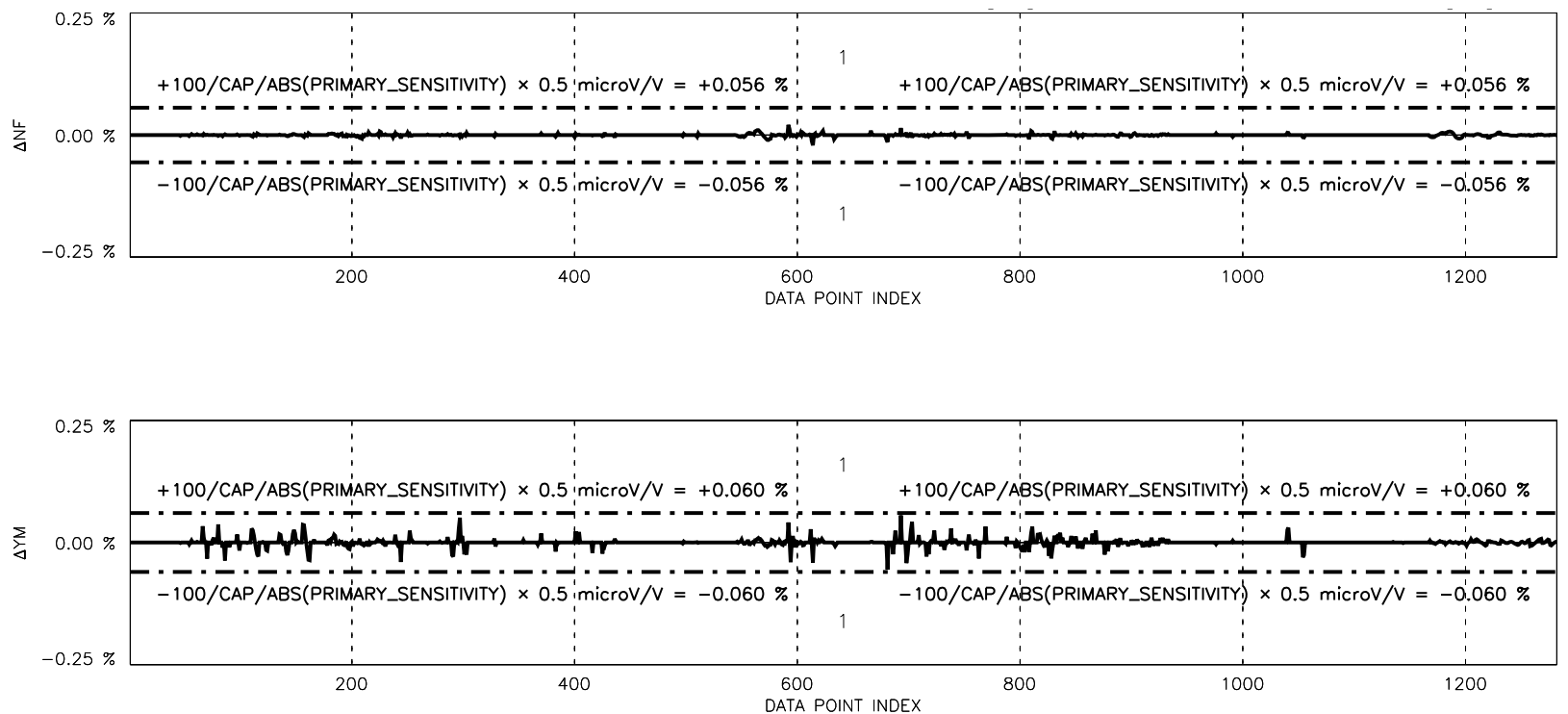

Fig. 5b Normal force and yawing moment repeat load differences for the MC400 balance calibration data (repeat load difference threshold $= \pm 100 /$ capacity $/ \mid$ sensitivity $\mid \times 0.5 \mathrm{microV} / \mathrm{V})$. 